\title{
Intervención educativa de enfermería en el nivel de conocimiento sobre VPH en estudiantes de una secundaria pública de Aguascalientes
}

\author{
Brito-Arenas Tessa Nívea*, Romo-Romero Maricruz*, Sánchez-Barbosa Miriam Alejandra*,
} Araiza-Hernández Martha Liliana**

Abstract

Objective:To identify the level of knowledge regarding the human papillomavirus (HPV) in public junior high school students after educational nursing intervention. Methods: This is a quantitative, quasi-experimental, and longitudinal study. A convenience non-probability sampling of 132 students of a public junior high school, with the prior informed consent of parents or guardians, was carried out. A 15-item instrument was applied to determine the knowledge of the respondents regarding HPV in students of a public junior high school in the state of Aguascalientes; a score of I 3-1 5 was interpreted as excellent, and I-3 as insufficient; there were a pre and post-intervention, having acceptable reliability with a Cronbach's alpha of 0.754 . The data and results were obtained using the SPSS Statistics program version 22.0-2013. Results: Before the educational intervention, $40.2 \%$ of the teenagers had a regular level of knowledge; after the educational intervention, $68.9 \%$ had an excellent level of knowledge. The ages (pre and posteducational intervention) of the population ranged from 13 to 15 years of age, $72.7 \%$ were 14 years old. Conclusions: By applying an educational intervention from the nursing staff to the teenagers of the public junior high school, improvement regarding the knowledge about the human papillomavirus was obtained. Nursing educational interventions on HPV favor changes in the attitudes and behavior in teenagers, impacting throughout their lives since ignorance of the consequences of unprotected sexual activity lead teenagers to engage in risky health behaviors. LUXMÉDICA, AÑO 15, NÚMERO 43, ENEROABRIL 2020, PP 71-78

Keywords: educational intervention, knowledge, human papillomavirus.

Estudiantes de la licenciatura de Enfermería del Centro de Ciencias de la Salud de la Universidad Autónoma de Aguascalientes. Aguascalientes, Ags., México.

** Profesora de Asignatura del Departamento de Enfermería del Centro de Ciencias de la Salud de la Universidad Autónoma de Aguascalientes.

Fecha de recibido: 30 de julio 2019

Fecha de aceptación: 30 de noviembre 2019

Correspondencia: LE. Martha Liliana Araiza Hernández. Departamento de Enfermería. Edificio 105. Centro de Ciencias de la Salud. Campus Universitario. Universidad Autónoma de Aguascalientes. Avenida Universidad \#940. Aguascalientes, Ags., México. Código postal 20131. Teléfono 4499108437. Correo electrónico: ilil1981@hotmail.com 


\section{Introducción}

El virus de papiloma humano (VPH) es la enfermedad de transmisión sexual más común en todo el mundo; 660 millones de personas lo presentan y causa infección en casi todos los hombres y mujeres en algún momento de sus vidas. En diversos estudios se ha descrito que, el virus de papiloma humano, puede ser causante de algunos tipos de cáncer en la mujer y en el hombre. El 99.7\% de los casos de cáncer cervicouterino se deben al (VPH) y, específicamente, el tipo 16 y 18; también pueden causar cáncer anal, de vagina, de vulva y orofaríngeo. El VPH es un virus que se contagia a través del contacto sexual vaginal, oral o anal con una pareja infectada con el virus del papiloma humano $y$, si bien el uso del preservativo reduce el riesgo del $65 \%$ al $85 \%$ de transmisión del $\mathrm{VPH}$, no lo elimina por completo. Lo anterior nos lleva a resaltar la importancia de disminuir la carga de la enfermedad mediante la prevención primaria. ${ }^{1}$

Según la Organización Mundial de la Salud (OMS), el cáncer cervicouterino $(\mathrm{CaCu})$ ocupa el cuarto lugar entre los tipos más comunes de cáncer que afectan a mujeres, con un número estimado de 266,000 muertes y 528,000 nuevos casos, según datos reportados en el 2012. ${ }^{2}$ La alta incidencia de infección por este virus está asociada al inicio temprano de la actividad sexual; de acuerdo con datos de la Secretaría de Salud, en 2014, se registraron 3,063 casos nuevos de $\mathrm{CaCu}$, con una tasa de incidencia de 6.08 por 100,000 habitantes. ${ }^{3}$ De acuerdo con los datos proporcionados por la Secretaría de Salud de la Ciudad de México, la infección por VPH ocupó el primer puesto con 7,353 personas infectadas. En Aguascalientes el Instituto de Servicios de Salud del Estado de Aguascalientes (ISSEA) reportó 47 casos en el año 2016. ${ }^{4}$

Aunque los factores de riesgo son múltiples, el principal es la infección por VPH; sin embargo, hay otros asociados, como tener múltiples parejas sexuales, el inicio temprano de relaciones sexuales, el tabaquismo y el nivel socioeconómico y educativo bajo. ${ }^{5} \mathrm{Al}-$ gunos autores como Goncalves $(2015)^{6}$ encontraron que de 4,325 adolescentes entrevistados en Brasil, el $18.6 \%$ de ellos habían iniciado su vida sexual antes de los 14 años. Mientras que en Cuba, el $62.6 \%$ había iniciado su vida sexual antes de los 16 años y sin protección en el $56.2 \%$ de los casos. ${ }^{7}$ Otros autores, como Dell Diana ${ }^{8}$ han reportado que hasta el $87 \%$ de la población adolescente entrevistada no escuchó hablar de VPH y el $80 \%$ tuvo un nivel bajo de conocimiento sobre VPH, mientras que García Rosique indicó que el $61.4 \%$ de las adolescentes ignoraban sobre el tema. ${ }^{9}$

El modelo de promoción de la salud de Nola Pender habla de un enfoque integral en el cuidado que debe tener la persona sobre su salud, con el objetivo de obtener cambios conductuales en el logro de estilos de vida saludable a través de la educación. Asimismo la autora menciona que "hay que promover la vida saludable que es primordial antes que los cuidados porque de ese modo hay menos 


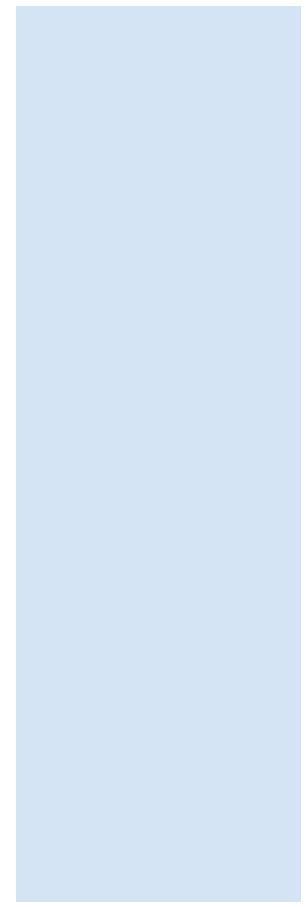

gente enferma, se gastan menos recursos, se le da independencia a la gente y se mejora hacia el futuro".${ }^{10}$ Una intervención educativa como método de enseñanza con enfoque preventivo debe "despertar el interés y atención de quien lo recibe, dándole valor a los contenidos del material brindado, motivando el interés de aprender, de estudiar y la satisfacción de cumplir las tareas que exige". ${ }^{8}$

Al respecto, Rodríguez y cols. recomiendan que abordar información vinculada con la sexualidad en adolescentes y jóvenes universitarios debe ir más allá de la presentación de un video o dar una conferencia, se debe procurar el aprendizaje significativo. ${ }^{9}$ Otros autores también proponen que dichas intervenciones educativas promuevan la información sobre comportamientos saludables y placenteros, y fortalezcan la capacidad de negociación asertiva y la toma de decisiones responsables. ${ }^{11,12}$ En otro estudio, realizado por Paredes ${ }^{13}$ sobre intervención educativa para elevar el nivel de conocimientos sobre cáncer de cuello uterino y el VPH, se reportó un nivel de conocimiento alto después de la intervención educativa. El objetivo de este estudio fue identificar el nivel de conocimiento sobre VPH en estudiantes de secundaria pública posterior a una intervención educativa impartida por personal de enfermería.

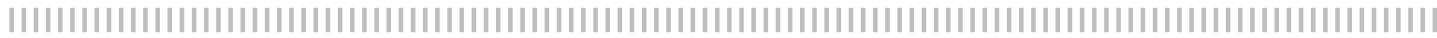

\section{Material y métodos}

Se realizó un estudio cuantitativo, cuasiexperimental, longitudinal no probabilístico, por conveniencia en estudiantes de tercer grado de una secundaria pública del estado de Aguascalientes, México, durante el periodo agosto-octubre de 2018. La población del estudio fueron 135 adolescentes que se encontraban cursando el tercer año de secundaria, de los cuales tres no aceptaron participar. La muestra final fue de 132 adolescentes. Se obtuvo el consentimiento informado tanto de padres o tutores, así como de los adolescentes. Se acudió a la institución educativa para la aplicación de los cuestionarios e intervención educativa.

El método empleado fue el cuestionario, para la recolección de datos se utilizó instrumento diseñado por Ortunio Calabres. ${ }^{14}$ Para la validación del instrumento en población mexicana, se realizó una prueba piloto en estudiantes de una secundaria pública de Aguascalientes y se obtuvo alfa de Cronbach 0,754. El instrumenro constó de 15 preguntas con respuestas cerradas, las cuales estuvieron dirigidas a determinar el conocimiento de los interrogados acerca del VPH en relación con la existencia del virus, su forma de transmisión, consecuencias, órganos afectados y medidas de prevención. El conocimiento se determinó mediante una escala de valoración siendo distribuido en las siguientes categorías: insuficiente: si respondía correctamente de 1-3 preguntas; regular. si respondía correctamente 4-6; bueno: si fueron 7-9 preguntas; muy bueno: si respondía de forma adecuada 10-12 preguntas $y$, excelente: si respondía correctamente de 13-15. La recolección de datos de la prueba piloto se realizó en otra secundaria pública que cumplió con los criterios de inclusión, durante la primera semana de agosto 2018.

Posteriormente se realizó la intervención educativa de enfermería con el programa de salud titulado "Virus del papiloma humano". La captura de datos se realizó en dos tiempos, el primero antes de realizar la intervención educativa de enfermería, el segundo después de la intervención. El 
análisis estadístico se realizó en el programa SPSS Statistics versión 22.0- 2013. Se realizó una prueba $T$ student para la comparación de medias, previa a la realización de la prueba de Kolmogov-Smirnov que dio como resultado una muestra con una distribución normal. El proyecto se sometió a la evaluación de un comité de investigación y se aprobó con el código AEI-50-18. En esta investigación no se considera de riesgo ya que solo se emplearon técnicas y métodos de investigación documental como cuestionarios o entrevistas.

Se protegió la privacidad del individuo sujeto de investigación, identificándolo solo cuando los resultados fueron requeridos y con previa autorización apegados a la Declaración de Helsinki y a la Ley General de Salud. ${ }^{15-16}$

\section{| | | | | | | | | | | | | | | | | | | | | | | | | | | | | | | | | | | | | | | | | | | | | | | | | | | | | | | | | | | | | | | | | | | | | | | | | | | | | | | | | | | | | | | | | | | | | | | | | | | | | | | | | | | | | | | | | | |}

\section{Resultados}

Los resultados corresponden a 132 adolescentes. La edad promedio, en la mayoría de los adolescentes, fue de 14 años (72.7\%), con un rango de 13 a 15 años. La distribución por sexo mostró un ligero predominio del sexo masculino con el $52.3 \%$ y el $47.7 \%$ correspondió al sexo femenino. El $22.7 \%$ de ellos manifestó haber iniciado su vida sexual a los 13 años y el $16.6 \%$ refirió que ha tenido más de una pareja sexual.

El nivel de conocimiento del VPH preintervención educativa de enfermería fue considerado regular y bueno en el $40.7 \%$ y $35.5 \%$, respectivamente (figura1). Es necesario resaltar que el $20.4 \%$ de ellos fueron calificados con un nivel insuficiente de conocimiento del VPH. En la evaluación pos-intervención, los resultados mostraron un conocimiento excelente y muy bueno en el $68.9 \%$ y $21.9 \%$, respectivamente, evidenciando el cambio tras realizar la intervención (figura 2$)(p=0.001)$.

En la pre-intervención, las respuestas que predominaron a la pregunta sobre si conocían las consecuencias de adquirir el virus del papiloma humano y los órganos afectados fueron no y no sé. Mientras que, en la pos-intervención educativa, el $43.18 \%$ de los hombres y el $43.18 \%$ de las mujeres manifestaron tener el conocimiento (figura 3 y 4 ).

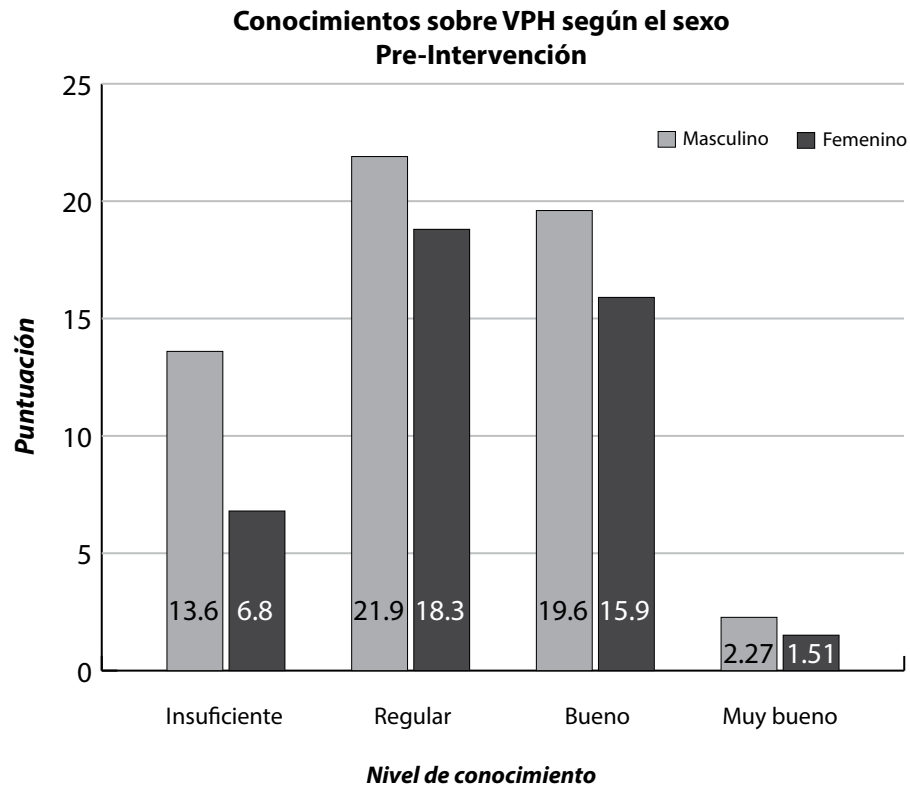

Figura 1. Distribución del nivel de conocimiento sobre VPH antes de la intervención educativa $(n=132)$ 


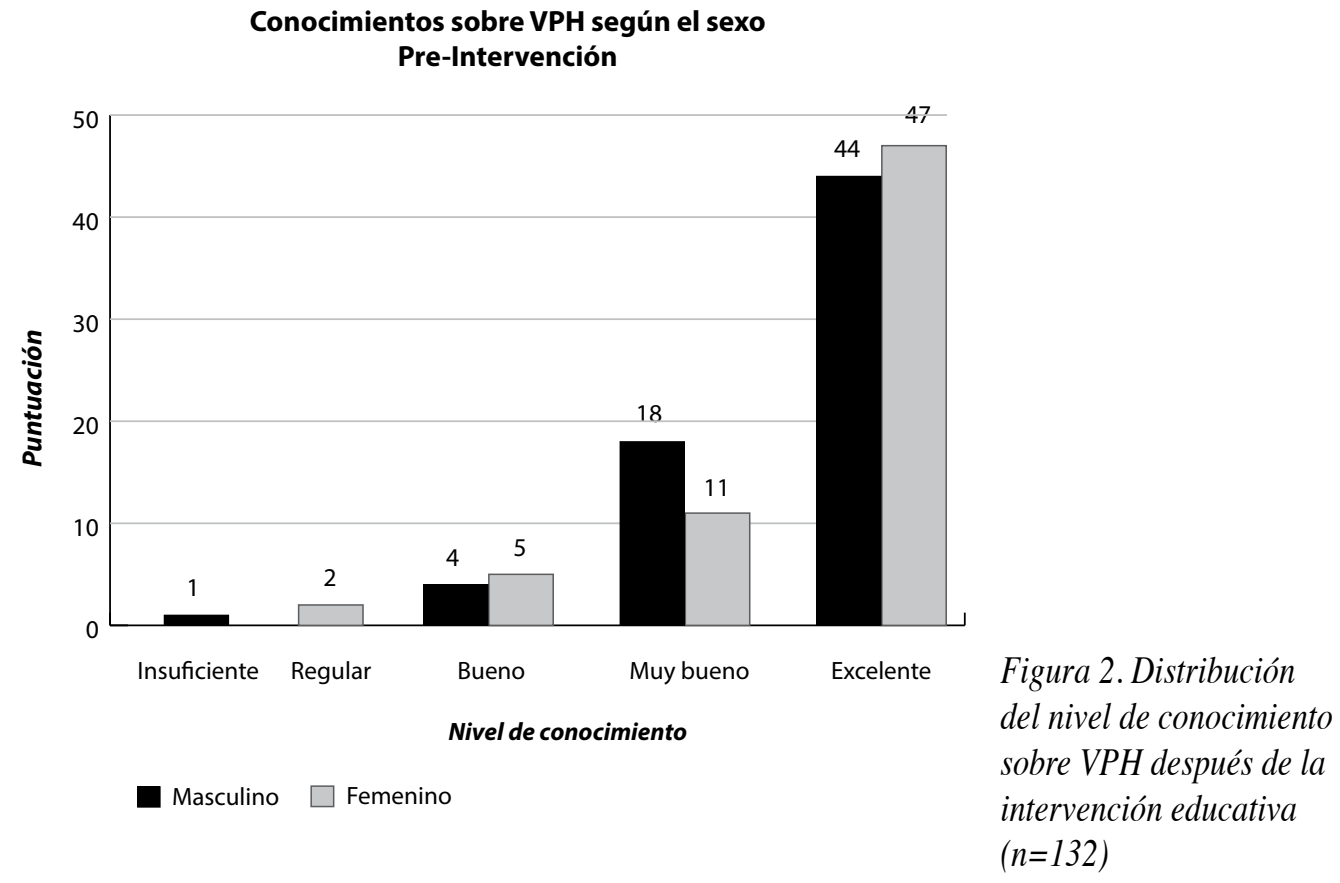

¿Puede provocar el virus del Papiloma Humano cáncer de vulva, cuello uterino y ano? Pre-intervención

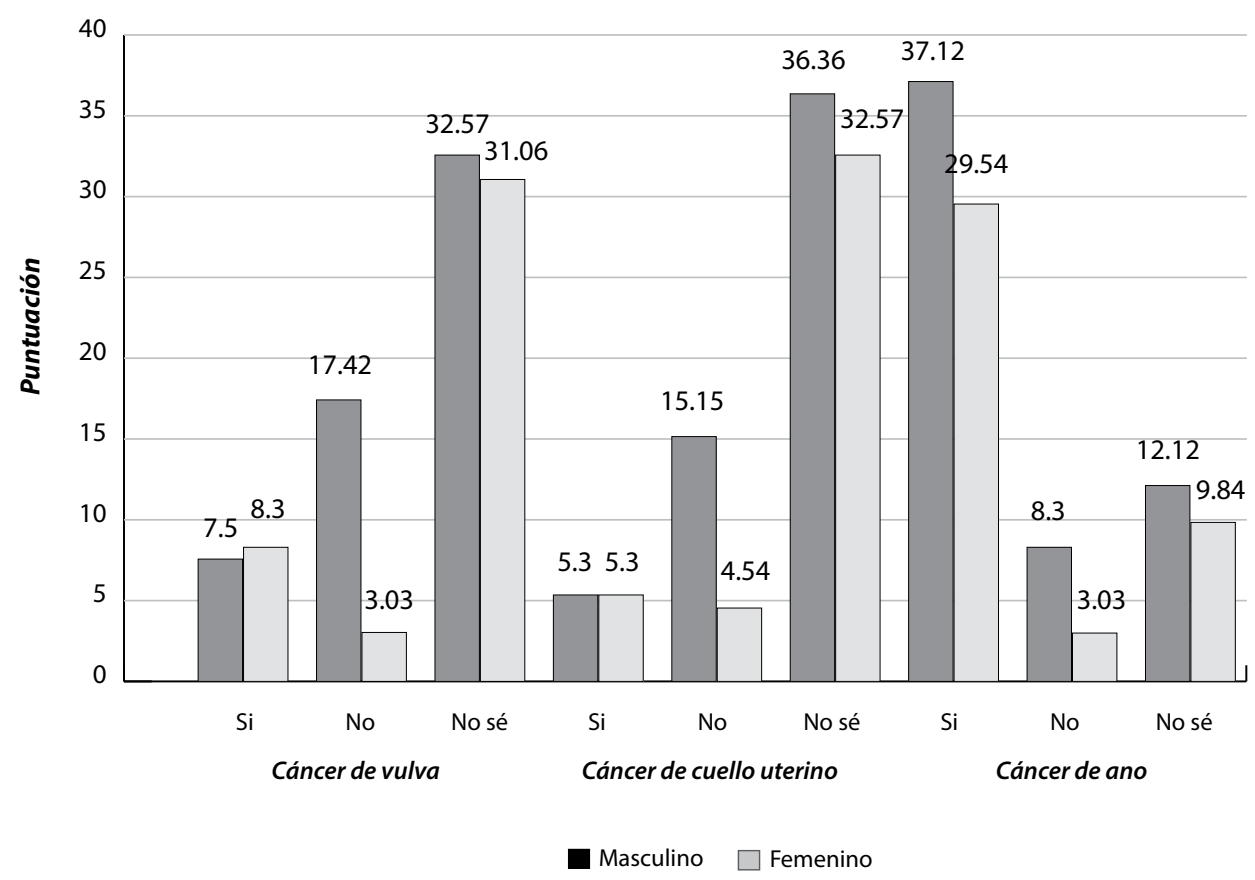

Fig.3 Fuente Estudiantes de una secundaria pública, Aguascalientes 2018 (n=132) 


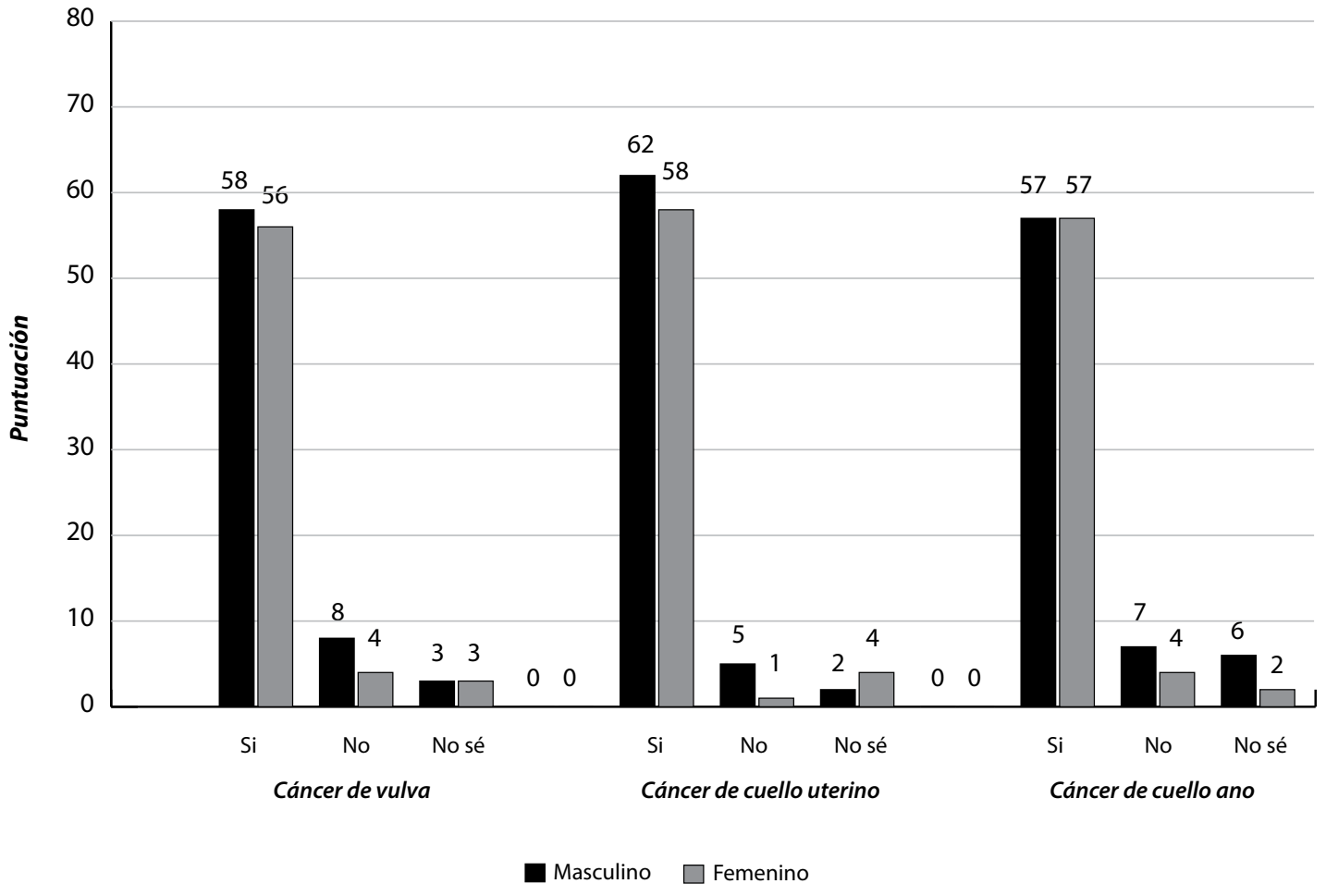

Fig.4 Fuente Estudiantes de una secundaria pública, Aguascalientes 2018 (n=132)

| | | | | | | | | | | | | | | | | | | | | | | | | | | | | | | | | | | | | | | | | | | | | | | | | | | | | | | | | | | | | | | | | | | | | | | | | | | | | | | | | | | | | | | | | | | | | | | | | | | | |

\section{Discusión}

En nuestro estudio se observó que, aproximadamente, uno de cada cinco de los adolescentes encuestados manifestó haber iniciado su vida sexual activa a los 13 años. Este hallazgo es similar a lo que han reportado otros autores como Goncalvez, ${ }^{6}$ y Puente Periñán. ${ }^{10}$ Resaltamos la importancia del inicio de la vida sexual activa temprana, ya que ha sido identificado como un factor de riesgo para la adquisición de una infección de transmisión sexual, entre los cuales se encuentra el VPH.

Por otro lado, se mostró un cambio significativo en los adolescentes de este estudio, entre el nivel de conocimiento sobre el VPH previo a la intervención y posterior a la misma; los adolescentes antes de la intervención se ubicaron en la categoría regular en conocimientos sobre el VPH. Posterior a la intervención, la mayoría de los adolescentes mostraron un gran cambio significativo respecto al conocimiento del VPH con la categoría excelente. Nuestros resultados son congruentes con lo reportado por otros autores como Dell, ${ }^{8}$ García Rosique, ${ }^{9}$ Paredes, ${ }^{13}$ Álvarez ${ }^{17}$ y Contreras Gutiérrez. ${ }^{18}$ Esto nos muestra la falta de conocimiento con la que se encuentran los adolescentes de la secundaria sobre aspectos básicos del virus de papiloma humano y resalta la importancia de proponer talleres educativos impartidos por el personal de enfermería a los adolescentes y jóvenes, procurando un aprendizaje significativo y que ayude a disminuir el inicio de su vida sexual activa y a fortalecer los conocimientos para la prevención de las consecuencias 
del virus de papiloma humano, como lo ha reportado Rodríguez y cols. ${ }^{11}$ Los resultados muestran la necesidad de atender un fenómeno que inicia desde la adolescencia, como también lo ha propuesto Campero y cols, ${ }^{12}$ es decir, intervenciones educativas en los diferentes instituciones escolares para promover la información saludable y orientar a los adolescentes a tomar decisiones asertivas.

\section{Conclusiones}

Se concluyó que, mediante la intervención educativa de enfermería, se generaron cambios significativos en el nivel de conocimiento sobre VPH en los adolescentes. El mayor cambio que predominó fue en relación a las formas de transmisión, consecuencias y órganos afectados.

Es necesario la participación de enfermería, así como de un equipo multidisciplinario de áreas afines, para realizar promoción a la salud a través de difundir información sobre el virus del papiloma humano para crear conciencia en los adolescentes y fomentar las relaciones sexuales con responsabilidad.

\section{Bibliografía}

1. Organización Mundial de la Salud [homepage en Internet]. Ginebra: OMS; 2017 [ actualizada 21 de agosto de 2017; consultado 10 Septiembre 2018]. Disponible en: http://www.who.int/immunization/ diseases/hpv/es/

2. Organización Mundial de la Salud. Estrategia mundial de prevención y control de las infecciones de transmisión sexual 2006-2015. Ginebra: OMS; 2007. Disponible en: http://apps.who.int/iris/bitstream/handle/10665/43773/9789243563473_spa. pdf? sequence $=1$

3. Secretaria de Salud [homepage en Internet]. México; 28 de Agosto 2016. Disponible en: https://www. gob. $\mathrm{mx} / \mathrm{salud} /$ articulos/semana-de-sensibilizacionen-cancer-de-cuello-uterino-2016-con-el-lemael-cancer-de-cuello-uterino-mascercadeloqueparece?idiom $=\mathrm{es}$

4. Puebla online [homepage en Internet]. Puebla; 2017. Disponible en: http://pueblaonline.com.mx/2015/ portal/movil/index.php/estado/item/5044868-casos-de-infeccion-por-papiloma-humano-enpuebla-en-lo-que-va-de-2017\#.WMvNfDyPPYU

5. José de Jesús Méndez Lira, Boletín UNAM- DGCS475, (Agosto, 2018) Disponible en: http://www. dgcs.unam.mx/boletin/bdboletin/2018_475.html

6. H. Goncalves, E.C. Machado, A.L. Soares, et al. Sexual initiation among adolescents (10 to 14 years old) and health behaviors Rev Bras Epidemiol., 18 (2015), pp. 1-19 Disponible en: http://dx.doi. org/10.1590/1. 980-5497201500010003 CrossRefView Record in Scopus
7. Raile AM,Tomey MA. Modelos y teorías en Enfermería. Barcelona: Elsevier; 2011 p.503-523.

8. Dell, D., Chen, H., Ahmad, M., \& Stewart, D. (2000). Conocimiento sobre el Virus del Papiloma humano entre los adolescentes. Revista de Obstetricia y Ginecología, 2000;96(5):653-656.

9. García Rosique RM, Torres Triana A, Rendón Quintero $M$. Conocimientos sobre prevención del cáncer cérvico-uterino en los adolescentes. Rev méd electrón [Seriada en línea] 2011;33(2). Disponible en URL:http://www.revmatanzas.sld.cu/revista \% 20 medica/ano\% 202011/vol2\% 202011/tema09.htm

10. Puente-Perpiñán Magalis, Haber-Reyes María del Pilar, Reyes-Losada Alina, Salas-Palacios Sara Ricci. Adolescentes e infección por virus del papiloma humano. Medisan 2014;18(6):769-775.

11. Rodríguez-Cabrera Aida, Sanabria-Ramos Giselda, Contreras-Palú Maria

Elena, Perdomo-Cáceres Belkis. Estrategia educativa sobre promoción en salud sexual y reproductiva para adolescentes y jóvenes universitarios Revista Cubana de Salud Pública 2013;39(1):161-174.

12. L. Campero-Cuenca, E.E. Etienzo, L. Suárez-López, Salud sexual y reproductiva de los adolescentes en México: evidencias y propuestas Gac Méd Mex, 2013;149:299-307.

13. Paredes Cruz E. Intervención educativa para elevar el nivel de conocimientos sobre cáncer de cuello uterino y el virus del papiloma humano en estudiantes de la UNASAN, filial Barranca, 2010 [Tesis]. Lima Perú. Universidad Nacional 29 Mayor de San Marcos, Facultad de Medicina Humana; 2012. Disponible en: http://cybertesis.unmsm.edu.pe/bitstream/ cybertesis/2433/1/Paredes_ce.pdf 
14. Ortunio Calabres Magaly Solmar, Guevara Rivas Harold, Herrera Leonett Edimar, Jiménez Rojas Karelis, Cardozo Castellano Rosa, Sánchez Pilimur Katherine. Conocimiento sobre el virus del papiloma humano en estudiantes de enfermería. Rev Obstet Ginecol Venez [Internet]. 2009;69(3):179-185. Disponible en: http://ve.scielo.org/scielo.php?script=sci arttext\&pid=S0048-77322009000300006\&lng=es.

15. Reglamento de la Ley General de Salud en materia de investigación en salud 2018 Título Tercero prestación de los Servicios de Salud, Titulo Quinto Investigación para la Salud, Titulo Séptimo Promoción de la Salud, Título Octavo Prevención y Control de Enfermedades y Accidentes. Última reforma publicada DOF 12-07-2018. Disponible en: http://www.diputados.gob.mx/LeyesBiblio/pdf/142_120718.pdf

16. HELSINKI DE LA AMM, "Declaración de Helsinki de la $A M M$ - Principios Éticos Para Las Investigaciones Médicas en Seres Humanos". Asociación Médica Mundial 2018. Disponible en: https://www.wma. net/es/policies-post/declaracion-de-helsinki-de- la-amm-principios-eticos-para-las-investigacionesmedicas-en-seres-humanos/. Ultima consulta 09 de noviembre del 2018.

17. Álvarez $L$, Peñuela $L$, Reyes $M$. Nivel de conocimiento de educación sexual, en adolescentes entre 14 y 19 años, de la Unidad Educativa "Domingo Hurtado". Comunidad de Nuevo Barrio. Barquisimeto Noviembre 1999-Marzo 2000 [Trabajo presentado como tesis de grado para optar por el título de Médico Cirujano]. Lara: Universidad Centroccidental "Lisandro Alvarado"; 2001

18. R. Contreras-González, A. Magaly-Santana, E.Jiménez-Torres, R.Gallegos-Torres, Á.XequeMoralesd, G.Palomé-Vegad, A.García-Aldecody, G.Perea-Ortíz. Nivel de conocimiento en adolecentes sobre el virus del papiloma humano. Enfermería universitaria [disponible en internet el 28 de febrero 2017]. [Consultado 20 de enero 2018]; 1-7. Disponible en: https://www.elsevier.es/es-revista-enfermeria-universitaria-400-articulo-nivel-conocimientos-adolescentes-sobre-el-S1665706317300155 